\title{
Toughening of Bisphenol-A Diglycidyl Ether-based Epoxy by Modification with Hydroxyl-terminated Liquid Natural Rubber
}

\author{
H. L. PHAM ${ }^{1 *}$, B. T. DO ${ }^{1}$, T. S. PHAM ${ }^{1}$ AND D. G. LE ${ }^{2}$
}

\begin{abstract}
Hydroxyl-terminated liquid natural rubbers (HTNRs), prepared by the Photo-Fenton reaction, were used to modify bisphenol-A diglycidyl ether-based epoxy (DGEBA). A chemical link between HTNRs and the epoxy resin was promoted employing toluene diisocyanate. The reactions between elastomers and epoxy resin were followed by FTIR. The mechanical properties of the composites were evaluated and the microstructure was investigated using scanning electronic microscopy. The results showed that the impact resistance of HTNR-modified DGEBA was superior to that of the pure epoxy resin. For the composites with HTNR, the impact resistance increased with elastomer concentration up to 2.5 parts per hundred parts of resin. Higher concentration of HTNR resulted in larger particles which gave lower impact values.
\end{abstract}

Key words: Natural rubber; liquid natural rubber; impact resistance; mechanical properties; epoxy resin; SEM; FTIR

Epoxy resins are one type of thermoset resins combining many attractive features such as high mechanical properties and excellent dimensional, thermal and environmental stabilities, toughness, rigidity, high temperature performance, chemical resistance, adhesive properties, formulation latitude and reactivity with a wide variety of chemical curing agents, high mechanical properties and excellent dimensional, thermal and environmental stabilities. Up till today, epoxy resins are widely applied as matrix of coatings, adhesives and composites. However, one practical disadvantage of highly crosslinked materials is it's poor resistance to crack propagation. These materials are brittle and cannot be used for damage tolerant applications due to the high cross-linking and inherent low toughness of epoxy resin. So, it is desirable to enhance their toughness without adverse influence on their other useful properties i.e. high thermal stability and low cure shrinkage (Kong et al. 2008; Thomas et al. 2070; Kaynak et al. 2002).

To overcome the above stated disadvantages, many types of modifiers were employed to improve the mechanical and thermal properties of epoxy resins, such as reactive liquid rubbers (Chikihi et al. 2002; Ratna \& Banthia 2007), amine-terminated poly(arylene ether ketone)s (Y1ldiz et al. 2027), triblock copolymers (Hydro \& Pearson 2007), thermoplastics (Goossens et al. 2006), carbon nanotubes (Moniruzzaman et al. 2006) and silicate-based nanofillers (Brunner et al. 2006). Modification with reactive liquid rubbers is one of the most effective strategies to toughen epoxy resins. In this new method, the liquid rubbers were

\footnotetext{
${ }^{1}$ Institute of Chemistry, Vietnam Academy of Science and Technology, 18-Hoang Quoc Viet Road, Cau Giay District, Hanoi, Vietnam

${ }^{2}$ University of Vinh, Nghe An, Vietnam

* Corresponding author (e-mail: huulyp@yahoo.com.vn)
} 
initially dissolved in epoxy resins and then the rubber separated as a discrete particulate phase due to the decreased solubility of rubber in matrices, this was caused by the increase of molecular weight of the cross-linked epoxy resins (Thomas et al. 2000). Many reactive liquid rubbers, such as acrylate rubber (Kong et al. 2008), carboxyl-terminated polybutadiene (CTPB) (Devi \& Srivastava 2007), carboxylterminated butadiene, acrylonitrile copolymer (CTBN) (Wise et al. 2000) and hydroxyl terminated polybutadiene (HTPB) (Ozturk et al. 2001) have been employed as effective toughening modifiers for epoxy resins. Other high performance polymers have also been used to modify epoxy resins, these include polysulphone (PSF), poly(etherimide) (PEI), polyimide (PI) (Mimura et al. 2001), poly(ether ether ketone) (Francis et al. 2005), polyetesulphone (PES) (Yang et al. 2008), amino-terminatd poly(arylene ether ketone) (Y1ldiz et al. 2007). Up to now, there have been no publications regarding the use of liquid natural rubbers (LNRs) in general and HTNRs in particular, to modify epoxy resins.

The main objective of the present work was to evaluate the use of HTNRs, as impact modifiers for DGEBA. The low reactivity of the hydroxyls of HTNRs toward the epoxy groups imposed the utilization of a route through the use of a bifunctional reagent that would react with both groups. The reaction of diisocyates with hydroxyls is well known in the formation of urethane linkages, as in the catalysed reaction of these with epoxy rings.

\section{EXPERIMENTAL}

\section{Materials}

Epoxy resin (ER), bisphenol-A diglycidyl ether-based epoxy (DGEBA), DER 331 trade name, from Dow Chemical Company Co. (USA), with equivalent weight of epoxy groups equal to $204.5 \mathrm{~g}$ / equiv, average molar ratio of secondary hydroxyl groups to epoxy groups equal to 0.355 , was dried at $80^{\circ} \mathrm{C}$ under vacuum for $3 \mathrm{~h}$ before use. The elastomer employed was a hydroxyl-terminated liquid natural rubber (HTNR) with $\mathrm{Mn}=3060$ containing hydroxyl groups and having an $\mathrm{OH}$ content of $2.2 \mathrm{~mol}$ of $\mathrm{OH} / \mathrm{mol}$ of HTNR. The toluene diisocyanate (Fluka AG) employed contained isomers 2,4 and 2,6 in 80:20 ratio). Piperidine was chosen as the curing agent (Aldrich, 98\%). Tetra-butyl ammonium iodide (TBAI) from BHD England of $98 \%$, was employed as the catalyst for the reaction between the epoxy and the isocyanate groups. Table 1 summarises the experimental conditions used to fabricate these DGEBA systems.

Table 1. Composition in parts per hundred of resin (phr) for each component in the final materials.

\begin{tabular}{c|c}
\hline Materials code & ER/ HTNR (phr) \\
\hline S0 & $100: 0$ \\
S1 & $100: 1.5$ \\
S2 & $100: 2.0$ \\
S3 & $100: 2.5$ \\
S4 & $100: 3.0$ \\
S5 & $100: 3.5$ \\
S6 & $100: 4.0$ \\
S7 & $100: 4.5$ \\
S8 & $100: 6.0$ \\
S9 & $100: 8.0$ \\
S10 & $100: 10.0$ \\
\hline
\end{tabular}

\section{Preparation of the Pre-polymer of DGEBA-TDI-HTNR}

The reactions to chemically modify HTNR and to subsequently link it to the epoxy resin were conducted in two steps, as shown in Figure 1. First, a reaction between HTNR and TDI was carried out to produce urethane links while leaving free isocyanate groups that would react afterwards with the epoxy groups of DGEBA. This second reaction was promoted upon the addition of the catalyst and increasing the temperature. At this stage a pre-polymer of HTNR and ER was formed in the epoxy resin medium. This pre-polymer would be further diluted before being cured, depending on the final HTNR concentration intended. The TDI 
was employed in excess of the equimolar ratio between NCO groups and OH groups in HTNR. This would leave enough isocyanate groups to react with epoxy groups. The modification of HTNR with TDI was conducted at $120^{\circ} \mathrm{C}$ for 3 $\mathrm{h}$ in a nitrogen atmosphere, in the presence of ER. After this period of time, the temperature was raised to $160^{\circ} \mathrm{C}$, while the catalyst TBAI was added, always in one part per hundred of ER. This condition was kept for another $3 \mathrm{~h}$ and after that the reaction mixture was cooled and allowed to rest at room temperature for $24 \mathrm{~h}$. Before initialization of the cure process, the pre-polymer was diluted by the addition of DGEBA and the composites with 1.5, 2.0, 2.5, 3.0, 3.5, 4.0, 4.5, 6.0, 8.0 and 10.0 p.h.r. of HTNR were prepared (S1 to S10 materials listed in Table 1).

\section{Cure Process}

The cure process of all materials prepared followed three steps: the mixture were degassed,
5 parts of piperidine were added and then the mixtures were degassed again. Subsequently the mixtures were poured into a pre-heated metal mould and cured in an oven at $120^{\circ} \mathrm{C}$ for $24 \mathrm{~h}$. Specimens for the tests were cut from this block of cured material.

\section{Characterization of Reaction Products}

The reaction products were characterized by infrared spectrometry with fourier transform (FTIR IMPACT 410, Germany) using $\mathrm{KBr}$ pellets.

Morphological characterization and the impact behaviour of cured materials was then studied. A minimum of 10 specimens of dimensions $63.5 \times 12.7 \times 10 \mathrm{~mm}$ were prepared and tested by the Izod impact method following the ISO 180-2000 procedure, in a universal machine for mechanical tests EMIC LTD model 202. For the morphological analysis, specimens were fractured after being cooled<smiles>CC(=CCC(C)(C)OO)CC(C)(C)O</smiles>

Hydroxyl-terminated liquid natural rubber-HTNR

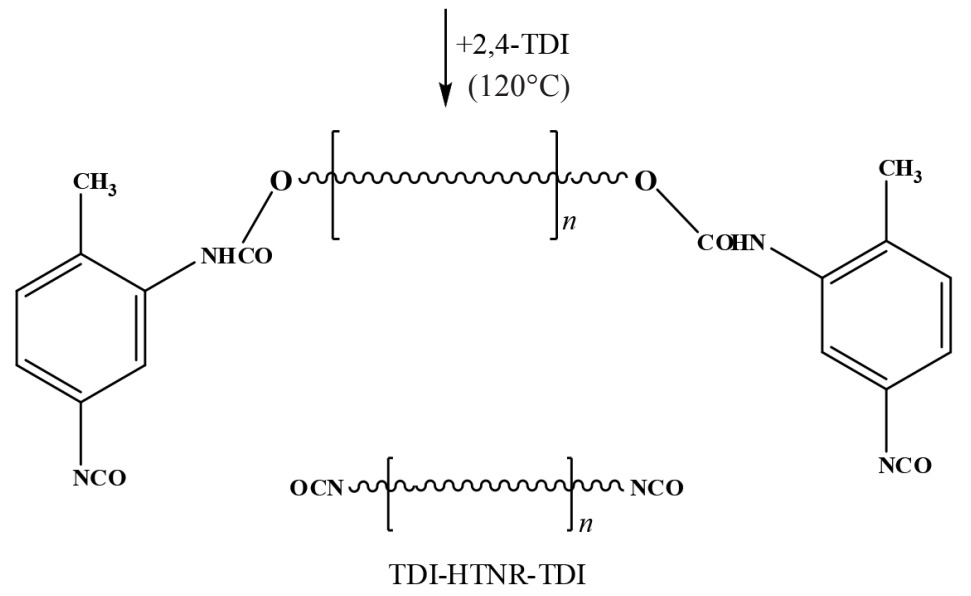

Figure 1. Scheme showing the formation of functional group urethane in the reaction between hydroxyl groups of HTNR and NCO group of TDI. 
in liquid nitrogen in order to prepare a smooth surface. The analysis was carried out in a scanning electron microscope, JEOL, JSM5300 (Japan) using $15 \mathrm{kV}$ working energy and a gold coating.

\section{RESULTS AND DISCUSSION}

\section{Characterization of the Pre-polymer of DGEBA-(TDI)-HTNR}

The process for the chemical modification of the DGEBA was carried out through two steps. The first involved the reaction of primary $\mathrm{OH}$ groups of HTNR with isocyanate groups of TDI, by employing an excess of TDI and a reaction temperature of $120^{\circ} \mathrm{C}$, in the presence of the DGEBA. In this step, a pre-polymer of TDI-HTNR-TDI with isocyanate terminated chains was formed, where TDI and HTNR were bound through urethane linkages, as illustrated in Figure 1. The FTIR of the product formed in the first step exhibited an absorption band at $1735 \mathrm{~cm}^{-1}$ (Figure $3 b$ ), indicating the formationn of the functional group urethane.

In the second step, the temperature was raised to $160^{\circ} \mathrm{C}$, the catalyst was added, and after $3 \mathrm{~h}$ the product formed presented the characteristic bands of oxazolidone besides the urethane bands, that is a carbonyl absorption at $1779 \mathrm{~cm}^{-1}$ attributed to a C-N link (Figure 3c). These FTIR results confirm the formation of the proposed links as shown in scheme 2 of Figure 2 but also reveal that DGEBA is linked to TDI-HTNR-TDI by the formation of two functional groups, respectively urethane and oxazolidone.

2

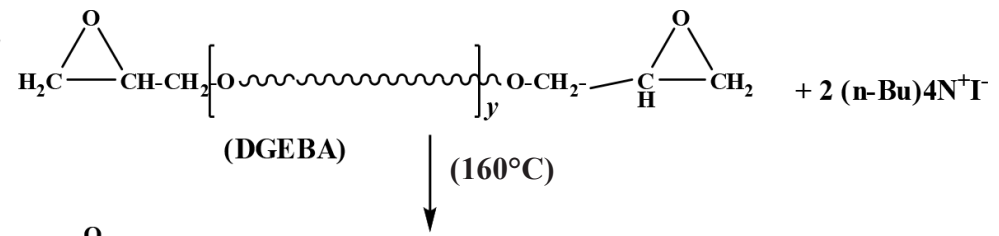

2
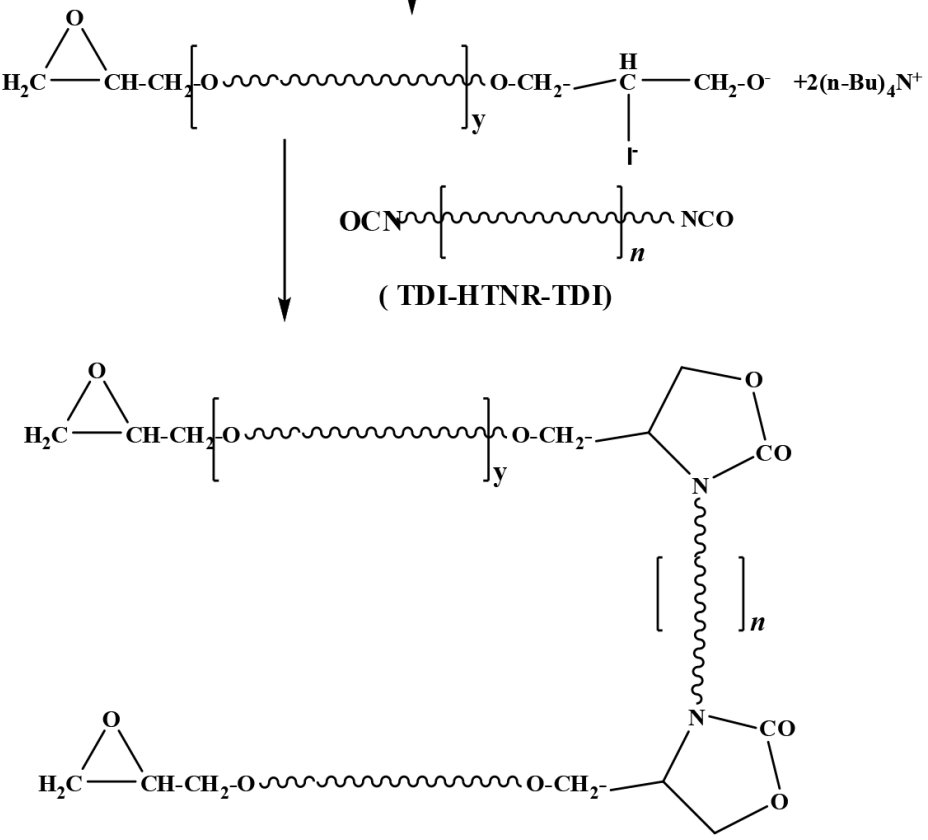

Figure 2. Scheme showing the formation of functional groups urethane and oxazolidone in the reaction between NCO groups of TDI-HTNR-TDI and epoxy groups of DGEBA. 


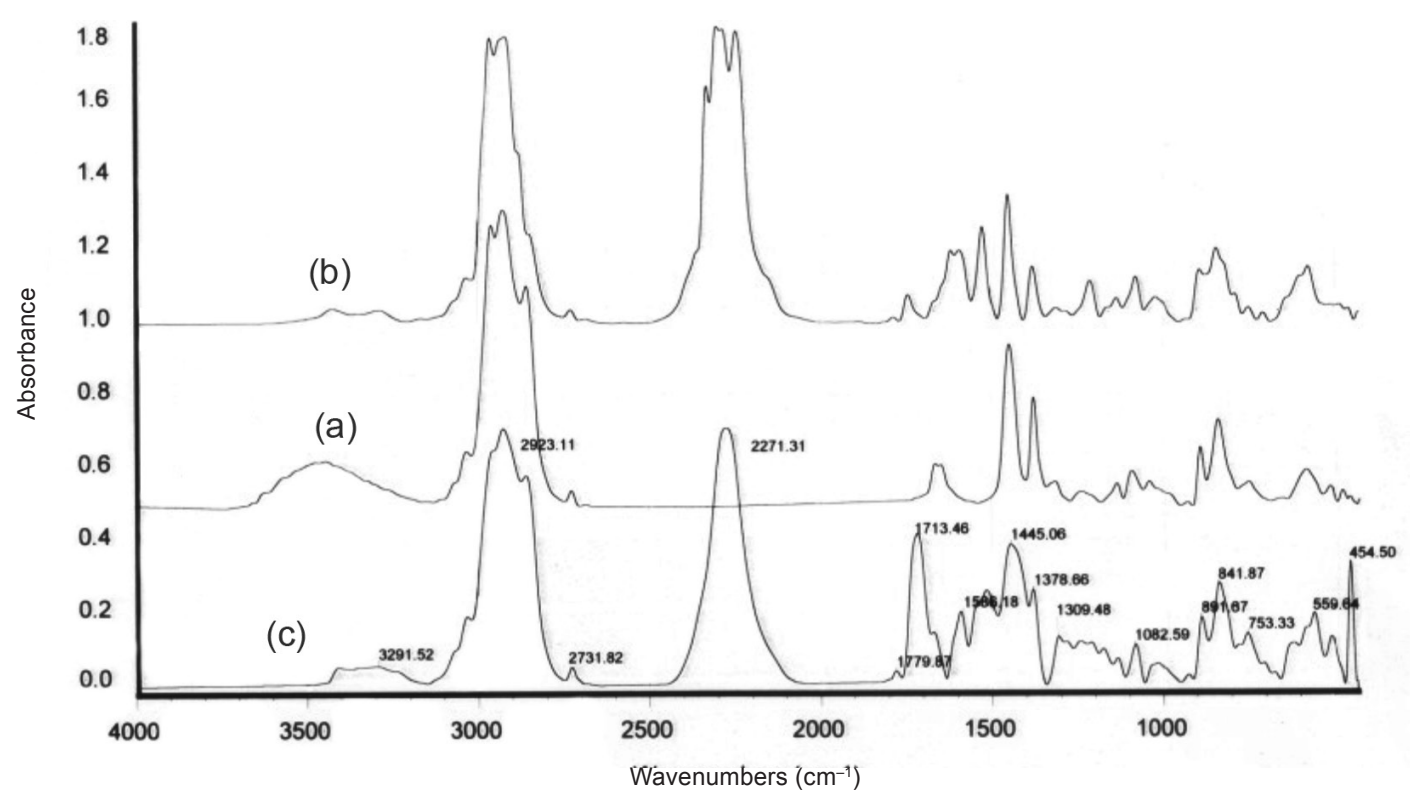

Figure 3. FTIR spectrum of HTNR: (a) TDI-HTNR-TDI; and the reaction product between TDI-HTNR$T D I$ (b) and DGEBA (c).

\section{Impact Strength}

The impact strength results are shown in Figure 4. The DGEBA composites were prepared with different HTNR concentrations. The impact strength of these materials increased with HTNR concentration, up to
$2.5 \mathrm{phr}$ but it decreased as more elastomer was incorporated. These results allow the conclusion that $2.5 \mathrm{phr}$ was the maximum content of HTNR that might be added in ER composites for a possitive effect upon the impact strength.

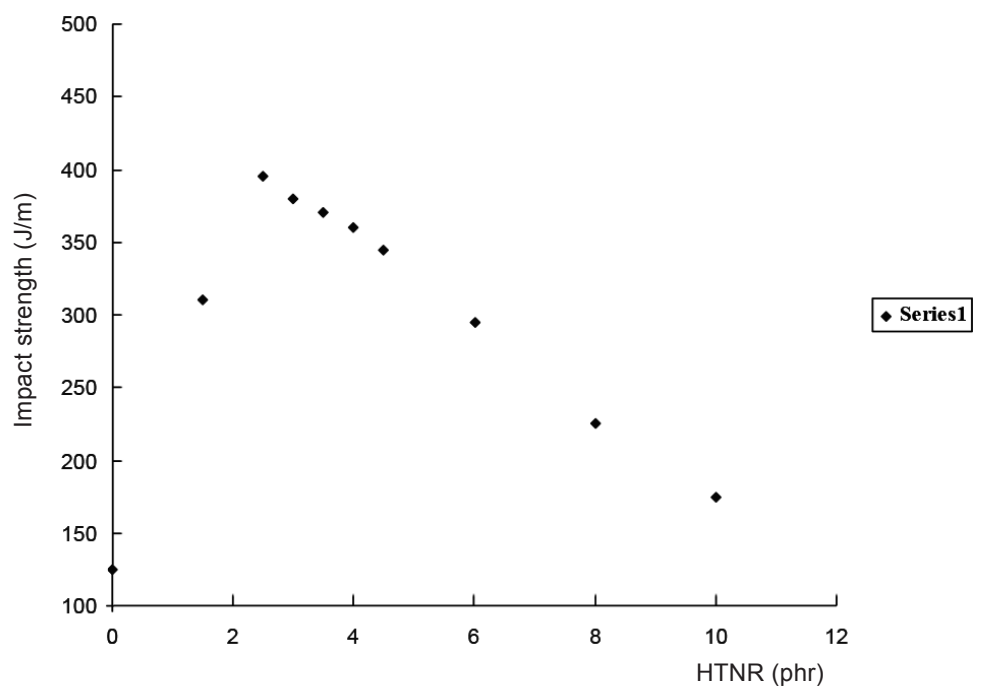

Figure 4. Impact strength of DGEBA modified with different HTNR concentrations. 


\section{Microstructure}

The microstructure of the composites were observed by the SEM to examine the correlation between particle sizes of the neat elastomeric phase and impact strength results. Figure 5 shows the SEM micrographs of neat DGEBA (a), DGEBA modified with $6 \%$ of HTNR (b), and DGEBA modified with $2.5 \%$ of HTNR.

From these, it could be seen that the unmodified ER had only one phase and the fracture surface was smooth. In the modification with 2 p.h.r. - 10 p.h.r. of HTNR, a two-phased morphology was seen with the elastomer-rich phase forming the continuous matrix and the elastomer-rich phase forming dispersed spherical particles, with rubber particles uniformly distributed throughout the matrix. The average particle diameters increased steadily with HTNR concentration. The data correlated with the impact strength results. As HTNR concentration increased, the impact strength decreased. All the materials presented phase separation between ER and elastome, which was necessary to improve impact resistance and also showed the excellent dispersion of the elastomeric particles in the epoxy matrix.

\section{Toughening Mechanism}

In the studied material, phase separation generated a morphology of spherical HTNR particles in the epoxy continuous phase, which lead to toughening mechanisms such as cavitation of rubber particles followed by void growth with consequent shear yielding of the epoxy matrix (Auad et al. 2001), the higher cavitation resistance of rubber particles might further improve the toughness of the structure (Pearson \& Yee 1993; Ratna \& Banthia 2001). The generation of the voids is due to the cavitation of rubber particles, which is the most important energy-dissipating mechanism in the case of rubber-toughened epoxy (Pearson \& Yee 1993; Ratna \& Banthia 2001). The reaction between NCO groups of TDI-HTNR-TDI and epoxy groups of DGEBA might lead to an increase in toughness of the epoxy network.

\section{CONCLUSION}

Hydroxyl-terminated liquid natural rubber (HTNR) was used to modify bisphenol-A diglycidyl ether-based epoxy (DGEBA). A chemical link between the HTNR and the epoxy resin was promoted employing toluene diisocyanate. The impact strength results of DGEBA modified with HTNR were superior to those of the pure epoxy resin. Microstructure studies employing SEM, clearly identified the two-phase nature of the rubber-modified epoxy, consisting of rubbery particles embedded in an epoxy matrix and also showed excellent dispersion of the elastomeric particles in the epoxy matrix. HTNR has the potential
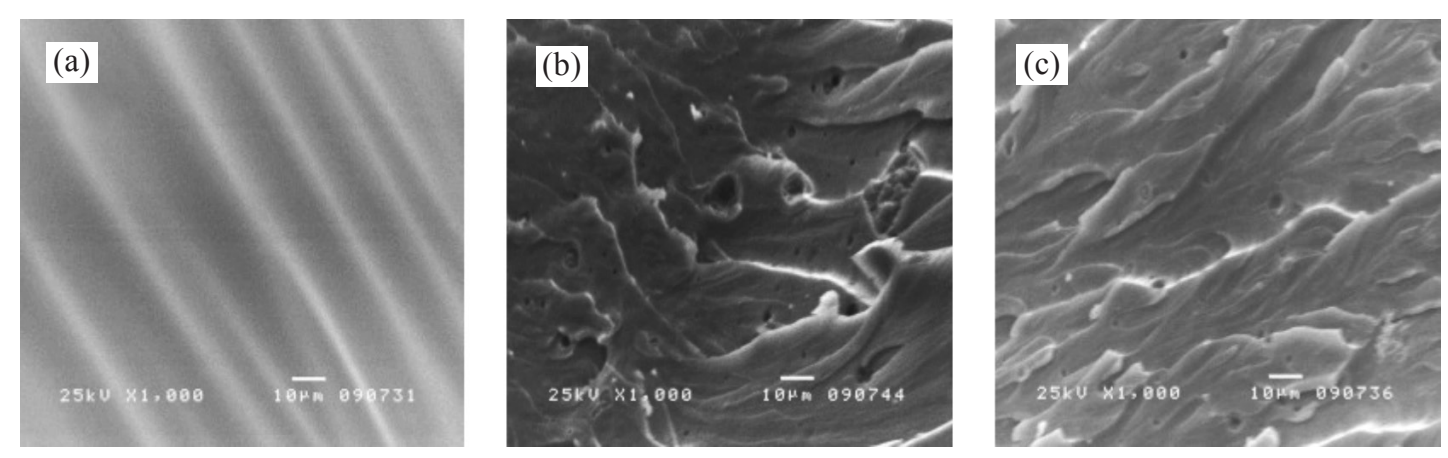

Figure 5. SEMs of the fracture surfaces of (a) neat DGEBA; (b) DGEBA modified with $6 \%$ of HTNR; and (c) DGEBA modified with 2.5\% of HTNR. 
to be a toughening agent for epoxy resins in general and DGEBA in particular, although the procedure used here might impose a limit to the amount of HTNR that could be incorporated.

\section{ACKNOWLEDGEMENT}

This study was supported by Vietnam's National Foundation for Science and TechnologyDevelopment (NAFOSTED, 104.04.70.09).

\section{Date of submission: April 2013 \\ Date of acceptance: March 2014}

\section{REFERENCES}

Auad, ML, Frontini, PM, Borrajo, J \& Aranguren, MI 2001, 'Liquid rubber modified vinyl ester resins: fracture and mechanical behavior', Polymer, vol. 42, pp. 3723-3730.

Brunner, AJ, Necola, A, Rees, M, Gasser, P, Kornmann, X, Thomann, R \& Barbezat, M 2006, 'The influence of silicate-based nano-filler on the fracture toughness of epoxy resin', Eng. Fract. Mech., vol. 73, pp. 2336-2345.

Chikihi, N, Fellahi, S \& Baker, M 2002, 'Modification of epoxy resin using reactive liquid (ATBN) rubber', Eur. Polym. J., vol. 38, pp. 251-264.

Devi, A \& Srivastava, D 2007, 'Studies on the blends of cardanol-based epoxidized novolac type phenolic resin and carboxyl-terminated polybutadiene (CTPB)', I. Mater. Sci. Eng. A, vol. 458, no. 1-2, pp. 336-347.

Francis, B, Thomas, S, Jose, J, Ramaswamy, R \& Rao, VL 2005, 'Hydroxyl terminated poly(ether ether ketone) with pendent methyl group toughened epoxy resin: miscibility, morphology and mechanical properties', Polymer, vol. 46, pp. 12372-12385.

Goossens, S, Goderis, B \& Groeninckx, G 2006, 'Reaction-induced phase separation in crystallizable micro- and nanostructured high melting thermoplastic/epoxy resin blends', Macromol, vol. 39, no. 8, pp. 2953-2963.

Hydro, RM \& Pearson, RA 2007, 'Epoxy- toughened with triblock copolymers', J. Polym. Sci., Part B: Polym. Phys., vol. 45, pp. 1470-1481.
Kaynak, C, Ozturk, A \& Tincer, T 2002, 'Flexibility improvement of epoxy resin by liquid rubber modification', Polym. Int., vol. 51, pp. 749-756.

Kong, J, Tang, Y, Zhang, X \& Gu, J 2008, 'Synergic effect of acrylate liquid rubber and bisphenol a on toughness of epoxy resins', Polym. Bull., vol. 60 , pp. 229-236.

Mimura, K, Ito, H \& Fujioka, H 2001, ‘Toughening of epoxy resin modified with in situ polymerized thermoplastic polymers', Polymer, vol. 42, pp. 9223-9233.

Moniruzzaman, M, Du, FM, Romero, N \& Winey, KI 2006, 'Increased flexural modulus and strength in SWNT/epoxy composites by a new fabrication method', Polymer, vol. 47, no. 1, pp. 293-298.

Ozturk, A, Kaynak, C \& Tincer, T 2001, 'Effects of liquid rubber modification on the behaviour of epoxy resin', Eur. Polym. J., vol. 37, no. 12, pp. 2353-2363.

Pearson, RA \& Yee, AF 1993, 'Toughening mechanisms in thermoplastic-modified epoxies: 1. Modification using poly(phenylene oxide)', Polymer, vol. 34, no. 17, pp. 3658-3670.

Ratna, D \& Banthia, AK 2007, 'Reactive acrylic liquid rubber with terminal and pendant carboxyl groups as a modifier for epoxy resin', Polym. Eng. Sci., vol. 47, no. 1, pp. 26-33.

Ratna, D, Banthia, AK \& Deb, PC 2001, ‘Acrylatebased liquid rubber as impact modifier for epoxy resin', J. Appl. Polym. Sci., vol. 80, pp. 1792-1801.

Thomas, R, Durix, S, Sinturel, C, Omonov, T, Goossens, S, Groeninckx, G, Moldenaers, P \& Thomas, S 2007, 'Cure kinetics, morphology and miscibility of modified DGEBA-based epoxy resin - effects of a liquid rubber inclusion', Polymer, vol. 48, pp. 1695-1710.

Wise, CW, Cook, WD \& Goodwin, AA 2000, 'CTBN rubber phase precipitation in model epoxy resins', Polymer, vol. 41, no. 12, pp. 4625-4633.

Yang, G, Zheng, B, Yang, J, Xu, G \& Fu, Sh 2008, 'Preparation and cryogenic mechanical properties of epoxy resins modified by poly(ethersulfone)', J. Polym. Sci. Part A: Polym. Chem., vol. 46, pp. 612-624.

Yıldız, E, Ozarslan, O, Kuyulu, A \& Güngör, A 2007, 'Toughening of epoxy resins by amine terminated poly(arylene ether ketone)s having pendant tertiary butyl groups', Polym. Bull., vol. 58, pp. 503-511. 